ANDREA WECHSLER PIZARRO

DEPARTAMENTO DE DISEÑO

FACULTAD DE ARQUITECTURA Y URBANISMO

UNIVERSIDAD DE CHILE

SANTIAGO, CHILE

ANDREAWECHSLER@UCHILEFAU.CL
Dossier RChD

\section{Materiales, Diseño y sustentabilidad}

\author{
Dossier RCHD \\ Materials, Design and sustainability
}

Este dossier de la revista RChD: creación y pensamiento presenta estudios originales sobre un tema relevante: materiales, diseño y sustentabilidad. Esta importancia se gesta a raíz del sistema lineal de producción y consumo actual, el que desemboca en un sobreconsumo de recursos y un abuso de los sistemas naturales para eliminar desperdicios. En otras palabras, el sistema de diseño, producción y consumo actual no considera el ciclo de vida completo de los materiales y los productos que estos conforman, con sus consecuencias negativas.

En nuestra sociedad, las grandes cantidades de residuos agrícolas, forestales, industriales, de posconsumo domiciliario o basura electrónica pueden generar serios problemas de contaminación y riesgos ambientales en su entorno. Según el Programa de las Naciones Unidas para el Medio Ambiente (PNUMA, 2012), los ecosistemas están amenazados debido a los impactos del desarrollo económico basado en el sobreconsumo de los recursos y el uso de los sistemas naturales como un recipiente de desperdicios, que afecta la viabilidad de los recursos renovables y los no renovables. Con el fin de darle una solución a este problema, se han propuesto distintas formas de abordarlo. Por ejemplo, el Programa para el Medioambiente de las Naciones Unidas (UNEP), desarrolló un marco de política general llamado "Consumo y Producción Sustentable" (SCP) en el año 2012. Este marco ha sido conformado por distintas visiones del consumo y producción, tales como la Ecología Industrial. Esta se define como "la ciencia del desarrollo sustentable" (Graedel \& Allenby, 1995). Es un campo de investigación multidisciplinario que combina aspectos de la Ingeniería, Economía, Sociología, Toxicología y las Ciencias Naturales. Estudia el flujo de materiales y energía a través de los sistemas industriales de todo el ciclo de vida de un producto con un particular interés en los impactos que las actividades industriales tienen en el medioambiente. Este concepto se enfoca además en las transformaciones y disipación del flujo de materiales y energías tanto en el sistema industrial como en sistemas naturales (Garner \& Keoleian, 1995). El impacto de cada producto sobre el medio ambiente debe considerar el ciclo de vida de los materiales y su proyección consciente, con la finalidad de evitar la acumulación de desechos y optimizar los recursos.

Según Odum \& Odum (1996; 1976), todos los sistemas naturales construyen un orden, se retroalimentan energéticamente y reciclan materiales. Estos sistemas reforzados se llaman "diseños que sobreviven". Estos pueden autosustentarse usando los resultantes del consumo para nutrir la producción de recursos que necesitan para sobrevivir, y así minimizar los desechos (Howard Thomas Odum, 1996). En contraste, un "diseño de sistema que no sobrevive" es lineal y no refuerza la producción con los resultados del consumo, dicho sistema es un ejemplo del patrón de consumo de la sociedad contemporánea. 
Existen marcos teóricos holísticos perfeccionados, para la selección de materiales y diseño para el ambiente construido basados en los conceptos de la Ecología Industrial. Algunos de estos trabajos son Construction Ecology (Kibert, Sendzimir, \& Guy, 2002) y Building Ecology (Graham, 2003). Ambas publicaciones promueven la práctica del pensamiento del ciclo de vida que incluye la consideración del sistema completo y efectos sobre el tiempo. Ambos autores aplican a los sistemas industriales analogías encontradas en ecosistemas naturales. En los sistemas naturales, los materiales funcionan en un ciclo cerrado donde ningún recurso es desperdiciado. Estas analogías integran los sistemas industriales y naturales para mejorar el uso de recursos en el ambiente construido.

Algunas de las estrategias usadas para reunir los requerimientos medioambientales de la Ecología Industrial son abogar por productos y materiales más durables, promover la selección apropiada de materiales, reducir la intensidad del material y adoptar una gestión de procesos eficientes (Garner \& Keoleian, 1995). Con el fin de promover una extensión de vida del material, la Ecología industrial recomienda especificar materiales reciclados y como último recurso, el uso de materiales reciclables para generar nuevos productos de ser necesario. El principio involucrado es el de convertir los residuos de un proceso en alimento de otro (McDonough \& Braungart, 2002). Una apropiada selección del material sugiere sustituir las materias primas de los materiales por unas que no sean tóxicas o que sean biodegradables. En relación a la estrategia de reducir la intensidad del material, se indica considerar el uso de recursos renovables. En cuanto a adoptar una gestión de procesos eficientes se sugiere incrementar la eficiencia energética de producción de un material o producto (Keoleian, Koch, \& Menerey, 1995); junto con aprovechar las distancias entre industrias cercanas, lo que promueve la localidad en las soluciones (Garner \& Keoleian, 1995; Grann, 1997).

Las diferentes perspectivas para definir los materiales compuestos dependen principalmente en la aplicación final o en el tratamiento que tendrá el material. Baillie (2004) sugiere que la responsabilidad ecológica consiste en evaluar qué es local y en entender la red de propiedades que se relacionan que debe informar el diseño responsable, manufactura y consumo. Otros consideran que un material es sustentable si es biobasado, o si consiste en biofibras y resinas de fuentes renovables y por lo tanto se espera que sea biodegradable (Mohanty, Misra, \& Drzal, 2002). Estos criterios deben sin duda ser definidos por cada uno de los diseñadores.

Si bien se han realizado investigaciones para desarrollar y refinar principios y marcos más sustentables para la producción y consumo de materiales, hasta el momento no ha habido tantos intentos de utilizar aquellos principios en aplicaciones concretas.

En resumen, las investigaciones presentadas son una oportunidad de generación de alternativas sustentables a la práctica actual para solucionar los problemas de sobreconsumo de recursos, abuso de sistemas naturales para eliminar desperdicios y el paradigma de un sistema de producción y consumo lineal en el ambiente construido.

Este dossier consta de ocho artículos. En el primer artículo, Ana Cravino y Jorge Pokropek se alinean con lo anteriormente planteado y presentan una reflexión 
sobre lo que implica un diseño ambientalmente consciente y la importancia de una responsabilidad ambiental y social en la toma de decisiones. En el segundo artículo, Andrés Valencia-Escobar, Alejandro Zuleta, Yuliana Areiza y Esteban Correa presentan el desarrollo de un marco de bicicleta plegable en madera de teca que se pueda integrar a una estrategia de movilidad sostenible.

Por otra parte, Sebastián Rodríguez, en su investigación, desarrolló un material biobasado en micelio de hongos descomponedores de madera Pleorotus ostreatus y Trametes versicolor y rastrojos de cereales y cáscaras de frutos secos. En su investigación, Rodríguez evaluó y caracterizó las propiedades físicas y mecánicas del material. El autor desarrolló a la vez una serie de propuestas formales que se podrían lograr con el crecimiento del material. En el cuarto artículo, Jenny Martel y Romina Pacheco plantean la conformación de un material compuesto basado en fibra de huira de mimbre en desuso, en el cual utilizan tecnología de baja complejidad. Se caracterizó el material, generando una ficha técnica y se investigaron posibles aplicaciones en el área del packaging secundario, para incorporar a los mismos habitantes de la zona. Los académicos Francisco Javier González Madariaga, Jamie Francisco Gómez Gómez y Luis Alberto Rosa Sierra generaron placas constructivas de menor impacto ambiental para particiones interiores o recubrir paramentos. Las placas consisten en un núcleo de un material compuesto de pastas de yeso y de los residuos de fibras secas de agave y partículas de plásticos expandidos. El recubrimiento de las placas es papel en ambas caras. Estos tableros se caracterizaron mecánicamente y se llevó a cabo una evaluación comparativa de ecodiseño.

En el sexto artículo, Daniella Parodi Miranda presentó los resultados de la investigación sobre el desarrollo de un material compuesto a partir del residuo de la agroindustria chilena cáscara de nuez Juglans regia. La autora identificó el compuesto como un material liviano con destacadas características de aislación térmica, moldeable y trabajable con herramientas comunes de la industria maderera; el material requiere de bajo nivel de tecnología y energía para ser procesado, genera una mínima cantidad de desperdicios y posee un ciclo de vida circular.

Romina Cayumil Montecino y Marcelo Adasme Valdés, por su parte, presentan un estudio sobre la problemática de la generación de e-waste a nivel mundial, y muestran alternativas de mejora para minimizar el impacto medioambiental asociado a su disposición. Las alternativas para abordar dicha problemática se encuentran dentro del concepto de economía circular, que considera no solamente el producto final, sino que también el diseño, proceso e impactos relacionados a su manufactura.

Finalmente, este dossier cierra con la investigación de Jimena Alarcón, Rodrigo García-Alvarado, Helena Aguilar y Paula Sánchez-Friera. En este estudio se buscó identificar la relación entre el color del vidrio y su eficiencia energética. Se experimentó con diversos luminóforos semitransparentes e incoloros, con el fin de lograr que las fachadas de energía pasiva de los edificios urbanos posean energía efectiva capturada a través de sus vidrios. Se llevó a cabo un estudio de aceptación usuaria basado en las emociones, en el cual se ponderó el grado de aceptación respecto de la presencia de color en el vidrio. 


\section{Referencias}

Baillie, C. (Ed.) (2004). Green Composites, Polymer Composites and the environment. Cambridge: Woodhead Publishing Limited.

Garner, A., \& Keoleian, G. A. (1995). Industrial ecology: an introduction. Ann Arbor, Michigan: National Pollution Prevention Center for Higher Education, University of Michigan.

Graedel, T., \& Allenby, B. (1995). Industrial Ecology. 1995. Prentice Hall, Englewood Cliffs, New Jersey, 7632, 83-187.

Graham, P. (2003). Building Ecology: First principles for a sustainable built environment (1 ed.). Oxford, Great Britain: Blackwell Science (UK).

Grann, H. (1997). The industrial symbiosis at Kalundborg, Denmark. The industrial green game. Implications for environmental design and management, 117-123.

Keoleian, G. A., Koch, J. E., \& Menerey, D. (1995). Life cycle design framework and demonstration projects: profiles of A TOT and AlliedSignal. Retrieved from

Kibert, C. J., Sendzimir, J., \& Guy, G. B. (2002). Construction ecology: nature as the basis for green buildings. London and New York: Taylor \& Francis.

McDonough, W., \& Braungart, M. (2002). Cradle to cradle: remarking the way we make things (1st ed. Vol. 1). New York: New York : North Point Press.

Mohanty, A., Misra, M., \& Drzal, L. (2002). Sustainable biocomposites from renewable resources: opportunities and challenges in the green materials world. Journal of Polymers and the Environment, 10(1), 19-26.

Odum, H. T. (1996). Environmental accounting: EMERGY and environmental decision making: John Wiley \& Sons.

Odum, H. T., \& Odum, E. C. (1976). Energy basis for man and nature (2 ed.). New York: McGraw-Hill.

UNEP. (2012). Global Outlook on SCP Policies: taking action together. Retrieved from Paris: http://www.unep.fr/scp/ go/pdf/UNEP_Global_Outlook_SCP_ExSum.pdf 